\title{
A Rare Cause of Precocious Puberty and Massive Ovarian Cysts Associated with Hypothyroidism
}

\author{
Nabil Moatassim Billah*, Hajar El Kouarty, Rachida Dafiri \\ Mohammed V-Souissi University, University Hospital Center Ibn Sina, Rabat, Morocco \\ Email: "moatassimbillah.nabil@gmail.com
}

Received 26 February 2016; accepted 22 March 2016; published 25 March 2016

Copyright $@ 2016$ by authors and Scientific Research Publishing Inc.

This work is licensed under the Creative Commons Attribution International License (CC BY). http://creativecommons.org/licenses/by/4.0/

(c) ()

\begin{abstract}
Van Wyk-Grumbach syndrome (VWGS) is characterized by juvenile hypothyroidism, delayed bone age, isosexual precocious puberty and massive ovarian cysts. We report such a case in a 7-yearold girl who presented with a bilateral pelvic pain, abdominal distension and signs of hypothyroidism. Physical examination showed a breast development and lack of pubic and axillary hair. There was no clitoromegaly. Pelvic ultrasonography and CT scan showed enlarged bilateral multiloculared multicystics ovarian masses. The pituitary MRI was recommended before an elevated prolactin, revealed a large sellar and suprasellar mass. The patient was started on thyroid replacement using $\mathrm{L}$-Thyroxine. Clinically, the patient's fatigue, pelvic pain, and abdominal distension are resolved. During the follow-up, her thyroid functions and serum prolactin normalized within 8 months. Ultrasonography revealed regression of ovarian cysts in 2 months measuring, with normal sized ovaries in 14 months.
\end{abstract}

\section{Keywords}

Van Wyk-Grumbach, Precocious Puberty, Hypothyroidism, Ovarian Cysts

\section{Introduction}

Van Wyk-Grumbach syndrome (VWGS) is characterized by juvenile hypothyroidism, delayed bone age, isosexual precocious puberty and massive ovarian cysts, with reversal to aprepubertal state following thyroid hormone replacement therapy [1]. This syndrome has been described since 1905, but the term Van Wyk and Grumbach syndrome was coined in 1960 [2]. In this entity, the hypothyroidism leads to growth delay with paradoxi-

${ }^{*}$ Corresponding author.

How to cite this paper: Moatassim Billah, N., Kouarty, H.E. and Dafiri, R. (2016) A Rare Cause of Precocious Puberty and Massive Ovarian Cysts Associated with Hypothyroidism. Open Journal of Clinical Diagnostics, 6, 1-5. 
cally and precocious puberty, while the primary hypothyroidism traditionally leads to both pubertal and growth delay. Undiagnosed, this syndrome can lead to unnecessary surgery of the ovaries, while all the symptoms may get sorted with thyroxine hormone replacement. We report such a case in a 7-year-old girl.

\section{Observation}

A 7-year-old female patient, presented to the department of pediatrics, with a bilateral pelvicpain, and abdominal distension. She had no urinary complaints or disorder of transit. The interrogation at that time, revealed a history of fatigue, excessive somnolence, deterioration in school performance, decreased activity, cold intolerance, constipation and weight gain. On physical examination showed a Breast development and lack of pubic and axillary hair, there was no clitoromegaly. There was no history of headache, vomiting, and visual symptoms.

She was referred to our department for an ultrasound exploration can explain its symptoms. Ultrasonography of the pelvis showed enlarged multiloculared multicystics ovaries (right ovary measuring $7.5 \times 12 \mathrm{~cm}$ and left ovary $7.7 \times 12.5 \mathrm{~cm}$ ), with a fine multiple septa andanechoic contents, there did not exist vegetations, neither of vascularization of the septa with doppler, nor of ascite. The uterus appeared postpubertal measuring $5.4 \times 3.2 \times$ $3.6 \mathrm{~cm}$ with an endometrial stripe of $12 \mathrm{~mm}$.

We completed by a breast and thyroid ultrasound which showed a hypoechoic heterogeneous thyroid gland and a decrease in his size (Figure 1). She had mild enlargement of both the breasts, with the breast tissue measuring $3 \times 2 \mathrm{~cm}$ (Figure 2).

Pelvic and abdominal CT scan showed bilateral multiloculated ovarian masses with cysticcomponents, with a fine multiple septa, and homogenous contents; the left measuring $8 \times 13 \mathrm{~cm}$ and the right $8 \times 12 \mathrm{~cm}$, Liver, spleen, pancreas and both kidneys were within normal range. There was no para-aortic lymph node enlargement and a normal urinary bladder (Figure 3).

Hormonal investigations revealed severe hypothyroidism (thyroid stimulating hormone (TSH) $165 \mathrm{mIU} / \mathrm{ml}$, free T4 $1.3 \mathrm{pmol} / \mathrm{L}$, thyroid antibodies negative), with elevated prolactin ( $\operatorname{Prl} 127 \mathrm{mcg} / \mathrm{L}$ ), follicle-stimulating hormone (FSH) was 7 IU/L and LH was 1 IU/L. Other laboratory investigations were normal (ACTH, cortisol,

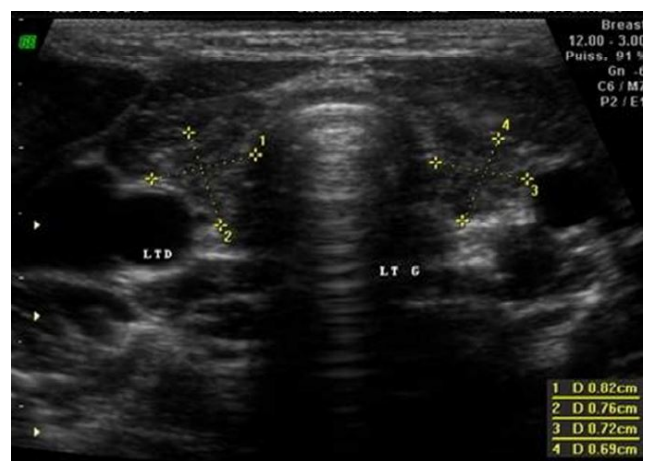

Figure 1. Thyroid ultrasound showed a hypoechoic heterogeneous thyroid gland and a decrease in his size.

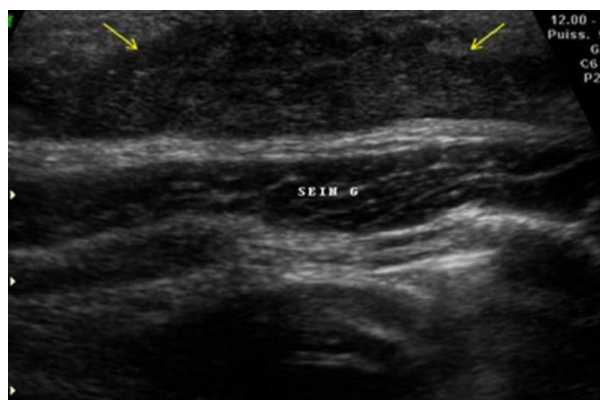

(a)

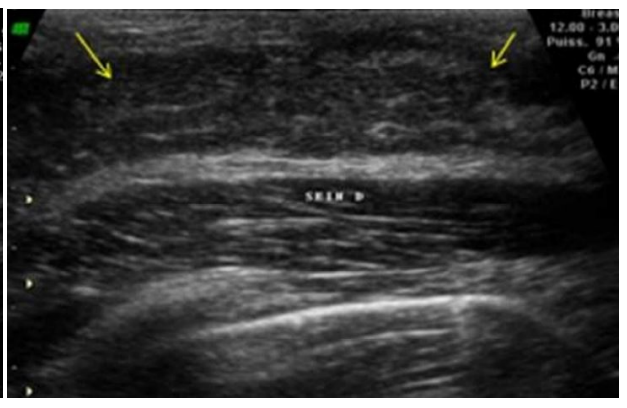

(b)

Figure 2. Brest ultrasound showed a mild enlargement of both the breasts, with the breast tissue measuring $3 \times 2 \mathrm{~cm}$. 


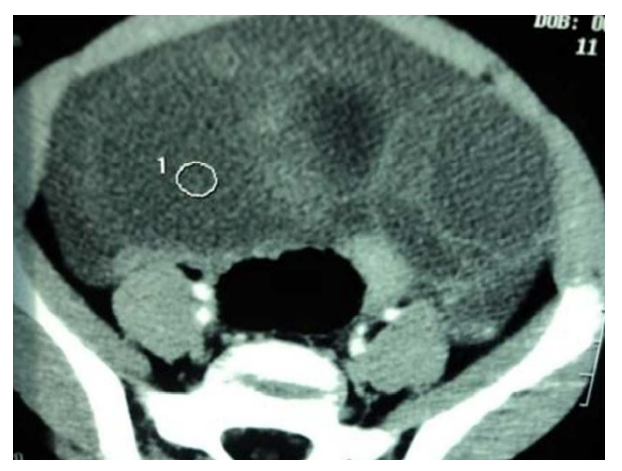

Figure 3. Pelvic and abdominal CT scan showed bilateral multiloculated ovarian masses with cystic components, with a fine multiple septa, and homogenous contents; the left measuring $8 \times 13 \mathrm{~cm}$ and the right $8 \times 12 \mathrm{~cm}$.

testosterone). Her bone age was delayed at 4 years (chronological age 7 yrs 4 mo).

The pituitary MRI was recommended before an elevated prolactin, revealed a large sellar and suprasellar mass of $12 \times 13 \times 18 \mathrm{~mm}$ size, that stretched the optic chiasm and respected thecavernous sinus, with isointense signal on T1-weighted sequences and hyperintense on T2-weighted imaging, and with homogeneously enhancing (Figure 4)

A diagnosis of Van Wyk and Grumbach syndrome was made, and the patient was started on thyroid replacement using L-Thyroxine. Clinically, the patient's fatigue, pelvic pain, and abdominal distension resolved.

During follow-up, her thyroid functions and serum prolactin normalized in 8 months. Repeat ultrasonography revealed regression of ovarian cysts at 2 months measuring (the left measuring $71 \times 33 \mathrm{~mm}$ and the right $60 \times$ $31 \mathrm{~mm}$ (Figure 5), with normal sized ovaries in 14 months.

\section{Discussion}

Van Wyk and Grumbach first reported multicystic ovaries with precocious puberty in patients with hypothyroidism [3]. The characteristic of this syndrome is that the primary hypothyroidism has classically been associated with delayed growth and delayed puberty in children, but in this syndrome it can be associated with precocious puberty.

This syndrome was more fully described by Van Wyk and Grumbach in 1960, Since then, sporadic reports have appeared in the literature about this condition [3] [4]. In the girl, the salient diagnostic features of this condition include a long-standing history of hypothyroidism with vaginal bleeding, and uncommonly with breast development or galactorrhea. Despite an early stage of puberty, there is a lack of pubic and axillary hair as was seen in our case.

The most common cause of hypothyroidism in these patients is autoimmune thyroditis [5]. Thyroid ultrasound examination showed a small dyshomogeneous and pseudonodular gland. The exact mechanism of the development of precocious puberty in VWGS remains speculative. Van Wyk and Grumbach believed that hormonal overlap in the negative feedback mechanism associated with hypothyroidism caused elevated gonadotropins [6] However, this does not explain the cases in the literature where FSH levels were found to be normal.

Given the inconsistent findings regarding FSH, a more accepted theory is the fact that TSH can act on the FSH receptor [7] [8]. Investigators have been divided over the role of PRL in the pathogenesis of the precocious puberty. Those who point to a central role for hyperprolactinemia contend that increased PRL levels are an important part of the syndrome [9]. They hypothesize that pituitary stimulation by thyrotropin-releasing hormone (TRH) leads to increased levels of TSH and PRL.

Sella turcica enlargement may be seen at times and it has been attributed to thyrotroph hyperplasia. Our case highlighted the role of MRI imaging in the diagnosis and follow-up of the thyrotroph hyperplasia in $\mathrm{PH}$ and the need for its early recognition to prevent the visual and neurological consequences of suprasellar mass and unnecessary surgery.

The multicystic ovaries may result from elevated levels of circulating gonadotrophins acting on the organ. The cysts resemble lutein cysts seen in Stein-Leventhal syndrome. It also is possible that increased sensitivity of 


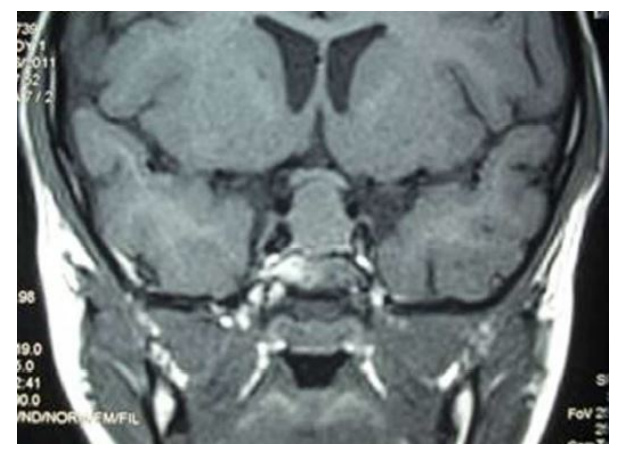

(a)

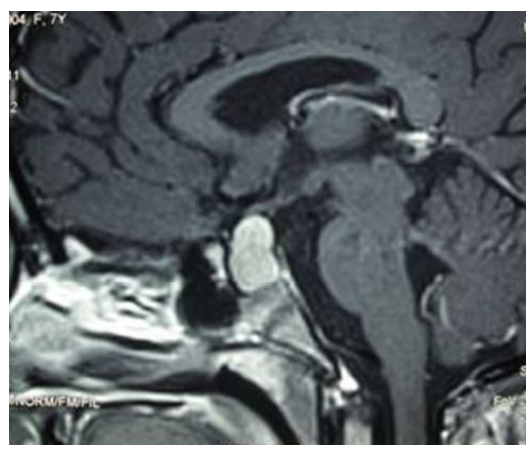

(b)

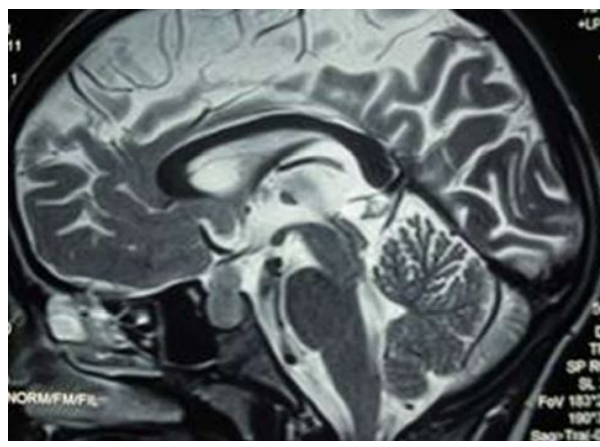

(c)

Figure 4. Pituitary MRI revealed a large sellar and suprasellar mass of $12 \times 13 \times 18 \mathrm{~cm}$ size, that stretched the optic chiasm and respected the cavernous sinus, that areisointense on T1-weighted sequences (a) and hyperintense on T2-weightedimaging (c), and with a T1-weighted MR homogeneously enhancing (b).

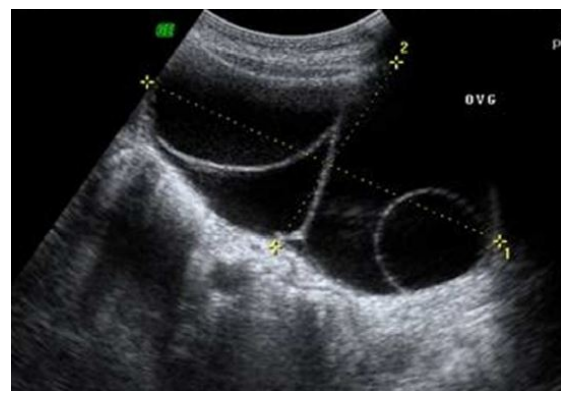

(a)

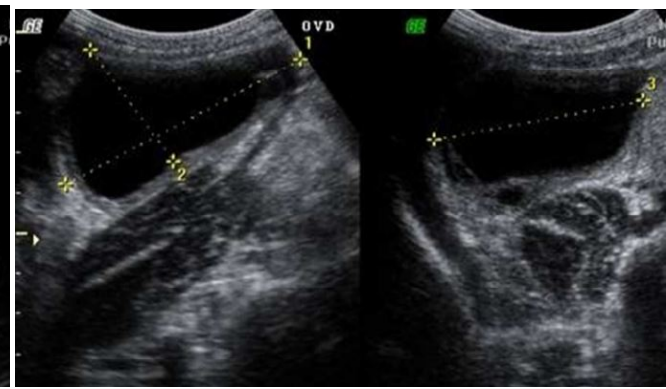

(b)

Figure 5. Ultrasonography revealed regression of ovarian cysts at 2 months measuring, the left measuring $71 \times 33 \mathrm{~mm}$ (a) and the right $60 \times 31 \mathrm{~mm}$ (b).

the ovaries to the circulating gonadotrophins could result from the hypothyroid state directly or via increased PRL [9].

The results of ultrasonography and CT showed bilateral multiloculated cystic ovarian masses, with benign characters; fine multiple septa, homogenous contents, without vegetations, neither of vascularization of the septa, nor of ascite. Thus, VWGS can be diagnosed nonoperatively, by the recognition of the salient clinical and imaging features, and appropriate confirmatory endocrine laboratory tests. Although there is little consensus regarding the precise etiopathogenesis of the disorder, the treatment approach is clear. All symptoms subside with thyroxine replacement, the endocrine abnormalities resolve, and even the ovarian cysts decrease in size or altogether disappear, as also in the present case during follow-up [10] [11].

\section{Conclusion}

In conclusion, we report the case of a young girl with the Van Wyk and Grumbach syndrome due to multicystic 
ovaries with precocious puberty and hypothyroidism. Recognition of one lesion of the triad should trigger an active search for additional lesions. Ultrasonography and MRI are suggested as the optimal modality for primary and follow-up investigations. The confirmation is done by an endocrine laboratory tests. All symptoms subside with thyroxine replacement, and the endocrine abnormalities are resolved.

\section{References}

[1] Van Wyk, J.J. and Grumbach, M.M. (1960) Syndrome of Precocious Menstruation and Galactorrhea in Juvenile Hypothyroidism. An Example of Hormonal Overlap in Pituitary Feedback. The Journal of Pediatrics, 57, 416-435. http://dx.doi.org/10.1016/S0022-3476(60)80250-8

[2] Durbin, K.L. and Diaz-Montes, T. (2011) Van Wyk and Grumbach Syndrome: An Unusual Case and Review of the Literature. Journal of Pediatric and Adolescent Gynecology, 24, e93-e96. http://dx.doi.org/10.1016/j.jpag.2010.08.003

[3] Hemady, Z.S., Siler-Khodr, T.M. and Najjar, S. (1978) Precocious Puberty in Juvenile Hypothyroidism. The Journal of Pediatrics, 92, 55-59. http://dx.doi.org/10.1016/S0022-3476(78)80070-5

[4] Lindsay, A.N., Voorhess, M.L. and MacGillivray, M.H. (1983) Multicystic Ovaries in Primary Hypothyroidism. Obstetrics \& Gynecology, 61, 433-437.

[5] Gordon, C.M., Austin, D.J., Radovick, S. and Lufer, M.R. (1997) Primary Hypothyroidism Presenting as Severe Vaginal Bleeding in a Prepubertal Girl. Journal of Pediatric and Adolescent Gynecology, 10, 35-38. http://dx.doi.org/10.1016/S1083-3188(97)70042-1

[6] Anasti, J.N., Flack, M.R., Froehlich, J., Nelson, L.M. and Nisula, B.C. (1995) A Potential Novel Mechanism for Precocious Puberty in Juvenile Hypothyroidism. The Journal of Clinical Endocrinology \& Metabolism, 80, 276-279.

[7] Barnes, N.D., Hayles, A.B. and Ryan, R.J. (1973) Sexual Maturation in Juvenile Hypothyroidism. Mayo Clinic Proceedings, 48, 849-856.

[8] Costin, G., Kershnar, A.K., Kogut, M.D., et al. (1972) Prolactin Activity in Juvenile Hypothyroidism and Precocious Puberty. Pediatrics, 50, 881-889.

[9] Chattopadhyay, A. and Kumar, V. (2003) Polycystic Ovaries, Precocious Puberty and Acquired Hypothyroidism: The Van Wyk and Grumbach Syndrome. Journal of Pediatric Surgery, 38, 1390-1392. http://dx.doi.org/10.1016/S0022-3468(03)00403-2

[10] Passeri, E., Tufano, A. and Locatelli, M. (2011) Large Pituitary Hyperplasia in Severe Primary Hypothyroidism. The Journal of Clinical Endocrinology \& Metabolism, 96, 22-23. http://dx.doi.org/10.1210/jc.2010-2011

[11] Hulse, A. (2014) Van Wyk-Grumbach Syndrome and Pituitary Hyperplasia in a Six-Year-Old Girl. Apollo Medicine, 11, 40-42. http://dx.doi.org/10.1016/j.apme.2013.10.009 